\title{
Relação entre os índices de maturação das vérte- bras cervicais e os estágios de calcificação dentária
}

Clarissa Lopes Vieira*, Ana Emília Figueiredo de Oliveira**, Cecília Cláudia Costa Ribeiro***,

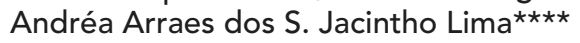

\section{Resumo}

Objetivo: avaliar a relação entre os diferentes índices de maturação óssea das vértebras cervicais e os estágios de calcificação dentária, bem como verificar as diferenças existentes entre os gêneros, em uma amostra de indivíduos de 10 a 14 anos de idade. Métodos: a amostra consistiu de telerradiografias laterais e radiografias panorâmicas de 464 indivíduos de ambos os gêneros, classificadas de acordo com os índices de maturação das vértebras cervicais descritos por Hassel e Farman (1995) e os estágios de calcificação descritos por Nolla (1960). Resultados: os resultados obtidos mostraram correlação entre as variáveis estudadas $(p<0,05)$. Conclusão: confirmando dados da literatura, observou-se uma precocidade, tanto nos índices de maturação das vértebras cervicais quanto nos estágios de calcificação dentária para o gênero feminino em relação ao gênero masculino.

Palavras-chave: Maturidade. Vértebras cervicais. Calcificação de dentes.

\section{INTRODUÇÃO}

O conhecimento do período de ocorrência do surto de crescimento puberal (SCP) é considerado vantajoso para os tratamentos ortodônticos que necessitem do uso de dispositivos influenciados pelo estágio de maturação do complexo craniofacial. Tal fase de crescimento também auxilia na elaboração do plano de tratamento, principalmente na indicação de procedimentos cirúrgicos ${ }^{7,12}$. O momento em que ocorre o SCP varia muito, tendo seu início, em média, entre os 10 e 12 anos, com seu pico ocorrendo geralmente 2 anos após o seu início e seu término por volta dos 15 anos de idade para o gênero masculino e dos 17 anos para o feminino ${ }^{30}$.

O estado de desenvolvimento de uma criança é melhor estimado considerando estágios específicos da maturidade fisiológica que, segundo a literatura, englobam 4 índices fisiológicos ou de desenvolvimento: maturidades somática, esquelética, dentária e sexual ${ }^{7,8}$. Dentre esses índices, a maturidade esquelética seria o índice mais confiável e mais frequentemente usado na rotina clínica.

A região da mão e punho vem sendo amplamente estudada e empregada como área de determinação da maturação óssea, sendo sua eficácia já cientificamente comprovada ${ }^{12,13}$. Nas últimas décadas, várias pesquisas vêm

\footnotetext{
* Mestre em Ciências da Saúde pela Universidade Federal do Maranhão - UFMA. Professora da disciplina de Radiologia e Imaginologia Oral da Universidade Federal do Maranhão - UFMA.

* Pós-doutora em Imagens Tridimensionais pela University of North Carolina (UNC / EUA). Professora da disciplina de Radiologia e Imaginologia Oral da Universidade Federal do Maranhão - UFMA.

*** Doutora em Cariologia pela Universidade Estadual de Campinas - UNICAMP. Professora da disciplina de Odontopediatria da Universidade Federal do Maranhão - UFMA

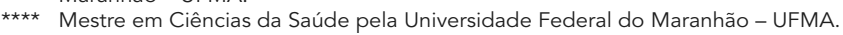


desenvolvendo e aperfeiçoando novos métodos para determinar a maturação óssea, sendo, atualmente, a análise das vértebras cervicais o método mais pesquisado $1,2,3,14,20,21,27$.

Segundo García-Fernandez et al. ${ }^{11}$, o uso das vértebras cervicais para determinar a maturidade esquelética ocorre desde a década de 70 , quando Lamparski ${ }^{18}$ analisou as mudanças morfológicas da segunda até a sexta vértebras cervicais e as classificou em seis estágios de maturação, comparando-os posteriormente com as modificações ósseas observadas na região da mão e punho. $O$ autor concluiu que as vértebras cervicais, como vistas nas telerradiografias laterais de rotina, foram tão estatística e clinicamente confiáveis na avaliação da idade esquelética quanto a técnica da mão e punho. García-Fernandez et al. ${ }^{11}$, no seu estudo, confirmaram tal afirmação de Lamparski. Em 1995, Hassel e Farman ${ }^{14}$ aperfeiçoaram o método estudado por Lamparski, analisando as mudanças morfológicas ocorridas somente da segunda até a quarta vértebra cervical, classificando-as em seis estágios de maturação, e também chegaram à mesma conclusão de Lamparski, assim como outras pesquisas realizadas posteriormente $\mathrm{e}^{21,27}$.

A maturidade dentária também é um índice fisiológico que vem sendo intensamente estudado para a predição do crescimento. Tal maturidade é identificada através da determinação da idade dentária, que pode ser avaliada de acordo com o número de dentes presentes na cavidade bucal ou com os estágios de calcificação de múltiplos dentes ou de elementos dentários individuais ${ }^{7}$. Silva ${ }^{29}$ afirmou que a utilização da idade dentária para a predição de crescimento seria ideal - por ser um dado prático e de fácil aplicação clínica -, pois a observação da irrupção dentária e dos estágios de desenvolvimento dos dentes é um dado de rápida assimilação e torna fácil a comunicação de ideias entre profissionais.

A existência de fortes relações entre os índices fisiológicos para a determinação do estado de desenvolvimento do indivíduo implicaria numa concordância dos seus mecanismos de controle, servindo aos profissionais no diagnóstico e plano de tratamento ${ }^{8}$. Já é bem estabelecido na literatura que existem fortes inter-relações entre as maturidades somática, esquelética e sexual ${ }^{8}$. Também é constatado que somente a idade cronológica não se constitui num índice confiável para a determinação do surto de crescimento puberal, pois essa sofre uma grande variação pela influência de fatores genéticos, raciais, ambientais, nutricionais, hormonais e sexuais ${ }^{13,22}$. Tratando-se da maturação dentária, ainda existem muitas controvérsias na literatura quanto à determinação da sua relação com os outros índices fisiológicos ${ }^{7,8,24,28}$, além de ainda não ter sido encontrado nenhum estudo que tenha analisado essa relação utilizando a maturação das vértebras cervicais como método para a determinação da maturação óssea.

Assim, considerando a importância do conhecimento do SCP e a grande contradição existente na literatura sobre a relação da maturidade dentária com outros índices fisiológicos, o objetivo deste estudo foi avaliar a relação entre os diferentes índices de maturação óssea das vértebras cervicais e os estágios de calcificação dentária, tendo como parâmetro a idade cronológica, bem como verificar as diferenças existentes entre os gêneros, em uma amostra de indivíduos com idades entre 10 e 14 anos.

\section{MATERIAL E MÉTODOS Seleção da amostra}

Após a submissão e aprovação deste estudo, foi possível executar a seleção da amostra, que foi constituída de telerradiografias cefalométricas em norma lateral e radiografias panorâmicas de pacientes, obtidas junto aos arquivos de alguns consultórios particulares de Ortodontia e cursos de especialização em Ortodontia de instituições públicas e particulares. Visando uma distribuição uniforme da amostra, pretendia-se obter 480 indivíduos, distribuídos em 8 grupos - de acordo com a faixa etária, variando de 6 em 6 meses, e de acordo com o gênero. Porém, a amostra foi constituída de 464 pacientes (228 do gênero masculino e 236 
TABELA 1 - Distribuição da amostra de acordo com a faixa etária e o gênero.

\begin{tabular}{|c|c|c|c|c|c|}
\hline \multirow{2}{*}{ grupo } & \multirow{2}{*}{ faixa etária } & \multicolumn{2}{|c|}{ telerradiografia } & \multicolumn{2}{|c|}{ panorâmica } \\
\hline & & masc. & fem. & masc. & fem. \\
\hline grupo 1 & 10 anos -10 anos e 6 meses & 25 & 26 & 25 & 26 \\
\hline grupo 2 & 10 anos e 7 meses -11 anos & 26 & 30 & 26 & 30 \\
\hline grupo 3 & 11 anos e 1 mês - 11 anos e 6 meses & 30 & 30 & 30 & 30 \\
\hline grupo 4 & 11 anos e 7 meses - 12 anos & 30 & 30 & 30 & 30 \\
\hline grupo 5 & 12 anos e 1 mês - 12 anos e 6 meses & 30 & 30 & 30 & 30 \\
\hline grupo 6 & 12 anos e 7 meses -13 anos & 30 & 30 & 30 & 30 \\
\hline grupo 7 & 13 anos e 1 mês - 13 anos e 6 meses & 30 & 30 & 30 & 30 \\
\hline grupo 8 & 13 anos e 7 meses - 14 anos & 27 & 30 & 27 & 30 \\
\hline SUBTOTAL & & 228 & 236 & 228 & 236 \\
\hline TOTAL GERAL & & & & & \\
\hline
\end{tabular}

do gênero feminino), com idades entre 10 e 14 anos, conforme mostra a tabela 1 .

\section{Critérios de seleção}

Foram selecionadas somente radiografias de boa qualidade, independentemente da raça, sendo que os pacientes não poderiam apresentar os seguintes critérios observados na ficha clínica:

- submissão a tratamento ortodôntico ou ortopédico prévio;

- doença ou síndrome que afetasse o crescimento;

- utilização precedente ou atual de medicamentos para o crescimento;

- nenhum tipo de anomalia (forma ou número) nos dentes a serem estudados, ou qualquer fator de impedimento da irrupção dos mesmos (dentes impactados por falta de espaço no arco, presença de patologias associadas à irrupção, etc.).

\section{Análise das radiografias}

As radiografias cefalométricas e panorâmicas foram analisadas utilizando-se metodologia cega por um examinador calibrado, de acordo com os critérios de maturação das vértebras cervicais e dos estágios de calcificação dentária, respectivamente, sendo a confiabilidade intraexaminador mensurada pelo coeficiente de Kappa $(\mathrm{k})^{19}$. Para tal, no início da pesquisa, foram realizadas duas análises consecutivas em 10\% da amostra, com intervalos de uma semana, nos quais a reprodutibilidade do examinador atingiu um valor de Kappa considerado excelente $(\mathrm{k}=0,87)$.

As radiografias foram classificadas de acordo com o índice de maturação das vértebras cervicais e o estágio de calcificação dentária, conforme o método de Hassel e Farman ${ }^{14}$ e o método de $\mathrm{Nolla}^{25}$, respectivamente. Importante ressaltar que a idade, o nome e o gênero do paciente, discriminados nas radiografias, foram vedados para que o examinador não fosse influenciado por essas características durante a inspeção radiográfica. Para a análise das radiografias, o examinador utilizou negatoscópio e lupa (magnificação de 3x).

\section{Método de Hassel e Farman}

O método de Hassel e Farman ${ }^{14}$ é construído sobre uma radiografia cefalométrica lateral, para a avaliação da morfologia da segunda, terceira e quarta vértebras cervicais (C2, C3 e C4) e, conforme as características observadas, permite a classificação em seis índices, como mostram o quadro 1 e a figura 1 .

\section{Método de Nolla}

O dente selecionado para o estudo foi o segundo molar inferior, levando-se em consideração a partir do estágio 7 de Nolla, por esse dente 


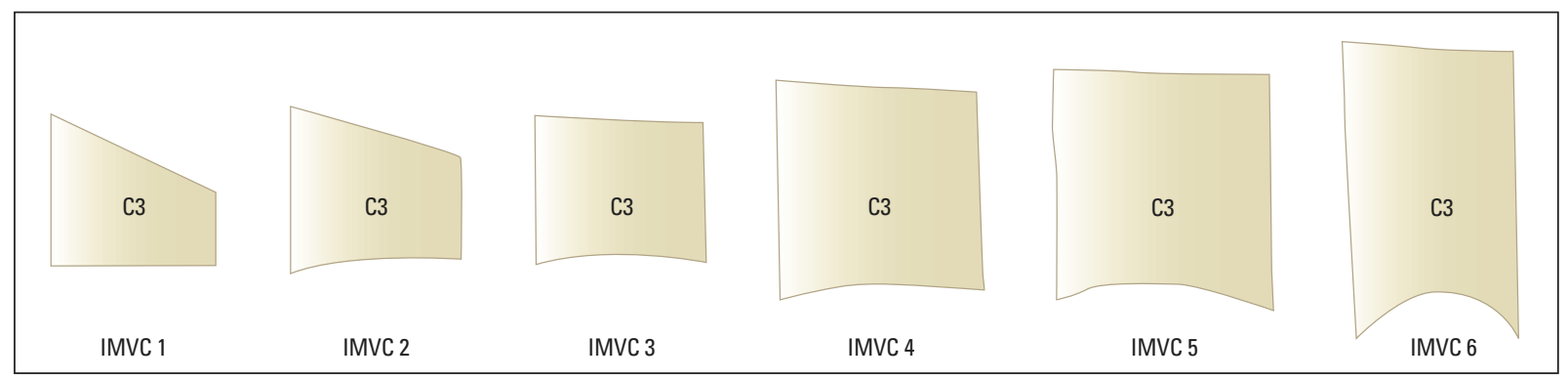

FIGURA 1 - Visão esquemática dos seis índices de maturação, considerando-se as características da vértebra C3 (Fonte: LIMA, 2004)20.

já se encontrar em formação radicular na faixa etária estudada. Esse elemento dentário foi escolhido, também, por ele ser facilmente visualizado em radiografias panorâmicas, devido à sua localização na região posterior da mandíbula ${ }^{25}$. A leitura dos estágios de calcificação dentária foi padronizada como sendo a do lado direito da radiografia e, em casos de dificuldade de avaliação, seria utilizada a avaliação do dente do lado oposto, pois, segundo Nolla ${ }^{25}$, não existe diferença estatisticamente significativa entre os lados direito e esquerdo no desenvolvimento dentário do indivíduo. A figura 2 demonstra uma visão radiográfica desses estágios.

\section{Registro dos dados e análise estatística}

Para o registro dos dados, foram elaborados dois tipos de formulários: um para o registro do índice de maturação das vértebras cervicais e o outro

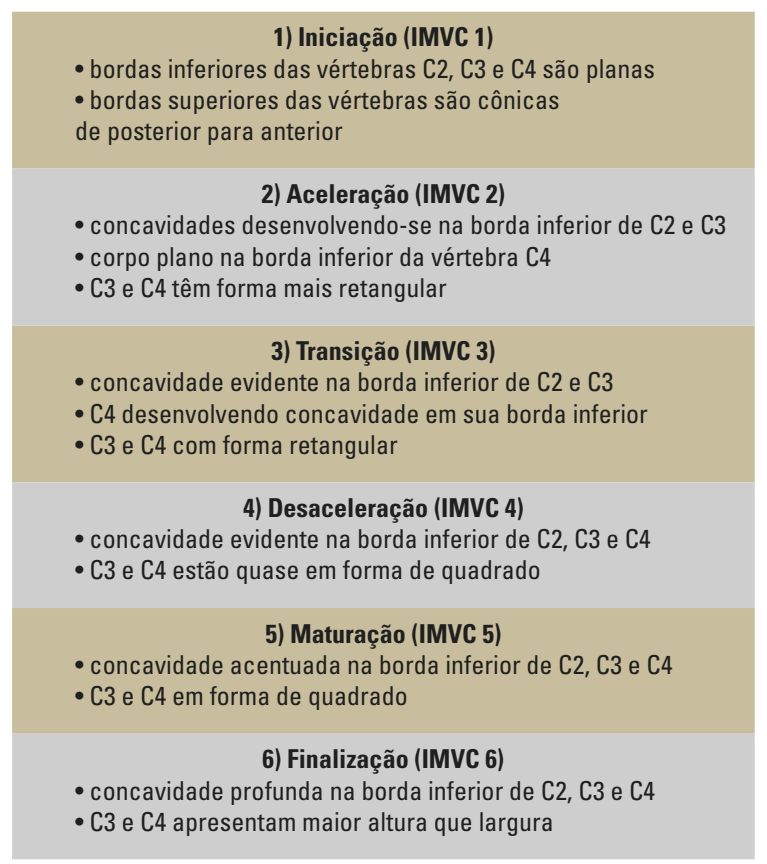

QUADRO 1 - Índices de maturação das vértebras cervicais, de acordo com Hassel e Farman ${ }^{14}$.

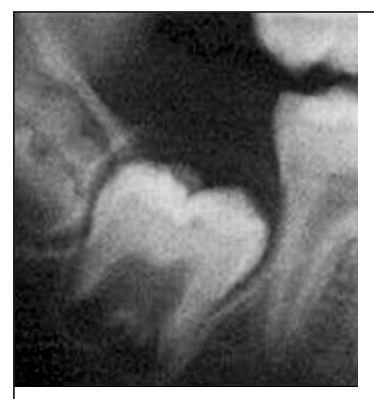

estágio 7 $1 / 3$ de raiz formado

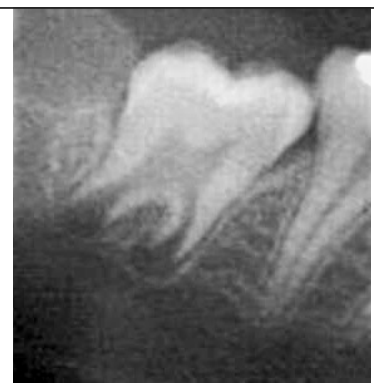

estágio 8 $2 / 3$ de raiz formados

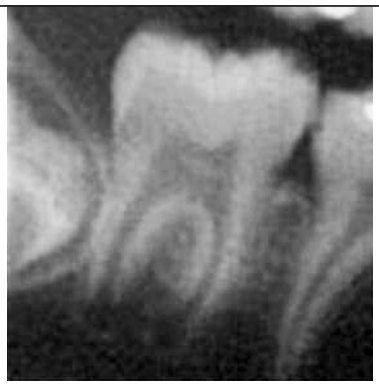

estágio 9 ápice aberto

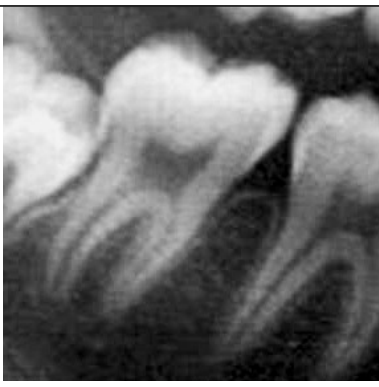

estágio 10 raiz completa

FIGURA 2 - Visão radiográfica dos estágios de Nolla considerados no estudo. 
para o registro do estágio de desenvolvimento dos segundos molares inferiores. Para cada grupo etário estudado, houve dois formulários de cada tipo, para enumerar os dados de acordo com o gênero.

Os dados obtidos foram submetidos à análise estatística utilizando o software Bioestat ${ }^{\circledR}$ versão 3.0 ${ }^{4}$. Para a verificação da existência de correlação entre os índices de maturação das vértebras cervicais e os estágios de calcificação dentária, foi empregado o coeficiente de contingência $C^{4}$. Para a verificação da existência de diferença estatisticamente significativa entre os gêneros, foi realizado o teste de Mann-Whitney ${ }^{4}$. Todos os resultados foram considerados significativos ao nível de significância de $5 \%(\mathrm{p}<0,05)$.

\section{RESULTADOS E DISCUSSÃO}

\section{Análise do surto de crescimento puberal}

A tabela 2 mostra a distribuição da amostra de acordo com os índices de maturação das vértebras cervicais, a idade e o gênero.

Considerando as afirmativas de Franchi, Bacceti e McNamara $\mathrm{Jr}{ }^{10}$, os resultados encontrados neste estudo revelaram que o pico de crescimento puberal ocorreu, com maior frequência, a partir dos 10 anos e 7 meses no gênero feminino, e a partir dos 12 anos e 7 meses no gênero masculino. Assim, tais dados demonstram que, na amostra estudada, o SCP se iniciou e atingiu o seu pico, com maior frequência, dois anos antes para o gênero feminino em relação ao gênero masculino, estando de acordo com registros da literatura ${ }^{23,30}$.

Para confirmar essa hipótese, foi aplicado o teste de Mann-Whitney nos diferentes grupos etários, comparando-os entre os gêneros, sendo encontrada diferença estatisticamente significativa em todos os grupos $(\mathrm{p}<0,05)$. Portanto, observou-se maior frequência da amostra em um índice mais avançado de maturação das vértebras cervicais no gênero feminino, como já está bem fundamentado na literatura. Carvalho ${ }^{5}$ também encontrou diferenças estatísticas entre os gêneros em relação à maturação óssea em crianças de 9 anos a 10 anos e 11 meses de idade. Segundo esse autor, tal diferença foi devida ao fato de que, nessa idade cronológica, as crianças estão muito próximas da adolescência, que certamente acontece mais cedo no gênero feminino.

\section{Análise dos estágios de calcificação dentária}

A tabela 3 mostra a distribuição da amostra de acordo com os estágios de calcificação, a idade e o gênero.

Com base nesses resultados, observou-se que o gênero feminino se mostrou em estágio mais avançado de formação dentária em relação ao gênero masculino, atingindo os estágios finais de calcificação mais precocemente. Aplicando-se o teste de Mann-Whitney, percebeu-se diferença estatisticamente significativa entre os gêneros somente nos grupos 1 e 2 ( $p=0,00$ e $p=0,03$, respectivamente). Nesses grupos, pode-se notar que no gênero feminino a maior frequência da amostra se distribuiu nos estágios 8 e 9 de Nolla, enquanto no gênero masculino a frequência da amostra foi maior nos estágios 7 e 8 . Nos outros grupos, a maior parte da amostra se concentrou no estágio 9 de Nolla, em ambos os gêneros. Também se pode perceber que, em ambos os gêneros, os indivíduos estudados conseguiram alcançar o estágio 9 de Nolla em um período de tempo menor que o período envolvido para mudar do estágio 9 para o estágio 10.

As diferenças nas idades cronológicas de ocorrência das várias fases de formação dentária encontradas entre os gêneros também foram confirmadas no estudo de Carvalho, Carvalho e Santos-Pinto ${ }^{6}$, no qual os autores evidenciaram o fato já conhecido de que o gênero feminino tende a ser mais precoce na cronologia de mineralização dentária ${ }^{24}$. Por outro lado, Kullman, Johanson e Akesson ${ }^{17}$ encontraram precocidade de calcificação dentária no gênero masculino, porém, esses autores utilizaram o terceiro molar inferior como elemento dentário de referência para a análise da cronologia de mineralização e, consequentemente, sua amostra se diferenciava também na faixa etária. 
TABELA 2 - Distribuição da amostra de acordo com o IMVC, a idade e o gênero.

\begin{tabular}{|c|c|c|c|c|c|c|c|c|}
\hline gênero & idade & $\begin{array}{c}\text { IMVC } \\
1 \\
\mathrm{n}(\%)\end{array}$ & $\begin{array}{c}\text { IMVC } \\
\mathbf{2} \\
\mathrm{n}(\%)\end{array}$ & $\begin{array}{c}\text { IMVC } \\
3 \\
n(\%)\end{array}$ & $\begin{array}{c}\text { IMVC } \\
4 \\
\mathrm{n}(\%)\end{array}$ & $\begin{array}{c}\text { IMVC } \\
\mathbf{5} \\
\mathrm{n}(\%)\end{array}$ & $\begin{array}{c}\text { IMVC } \\
\mathbf{6} \\
\mathrm{n}(\%)\end{array}$ & $\begin{array}{l}\text { total } \\
\mathrm{n}(\%)\end{array}$ \\
\hline \multirow{8}{*}{ masculino } & $10 a-10 a 6 m$ & $10(40,0)$ & $14(56,0)$ & $1(4,0)$ & - & - & - & $25(100)$ \\
\hline & $10 a 7 m-11 a$ & $7(26,9)$ & $14(53,8)$ & $5(19,3)$ & - & - & - & $26(100)$ \\
\hline & $11 \mathrm{a} 1 \mathrm{~m}-11 \mathrm{a} 6 \mathrm{~m}$ & $4(13,3)$ & $19(63,3)$ & $7(23,4)$ & - & - & - & $30(100)$ \\
\hline & $11 a 7 m-12 a$ & $8(26,7)$ & $19(63,3)$ & $3(10,0)$ & - & - & - & $30(100)$ \\
\hline & $12 a 1 m-12 a 6 m$ & $2(6,7)$ & $17(56,7)$ & $11(36,6)$ & - & - & - & $30(100)$ \\
\hline & $12 a 7 m-13 a$ & $3(10,0)$ & $10(33,4)$ & $11(36,6)$ & $4(13,3)$ & $2(6,7)$ & - & $30(100)$ \\
\hline & 13a1m-13a6m & $2(6,7)$ & $9(30)$ & $12(40)$ & $5(16,6)$ & $2(6,7)$ & - & $30(100)$ \\
\hline & $13 a 7 m-14 a$ & - & $8(29,6)$ & $13(48,2)$ & $4(14,8)$ & $2(7,4)$ & - & $27(100)$ \\
\hline \multirow{8}{*}{ feminino } & $10 a-10 a 6 m$ & $2(7,7)$ & $12(46,1)$ & $11(42,3)$ & $1(3,9)$ & - & - & $26(100)$ \\
\hline & $10 a 7 m-11 a$ & $4(13,3)$ & $10(33,3)$ & $15(50,0)$ & $1(3,4)$ & - & - & $30(100)$ \\
\hline & $11 \mathrm{a} 1 \mathrm{~m}-11 \mathrm{a} 6 \mathrm{~m}$ & $5(16,7)$ & $7(23,3)$ & $13(43,3)$ & $5(16,7)$ & - & - & $30(100)$ \\
\hline & $11 a 7 m-12 a$ & - & $11(36,6)$ & $9(30,0)$ & $9(30,0)$ & $1(3,4)$ & - & $30(100)$ \\
\hline & $12 \mathrm{a} 1 \mathrm{~m}-12 \mathrm{a} 6 \mathrm{~m}$ & $1(3,3)$ & $4(13,4)$ & $13(43,4)$ & $10(33,3)$ & $1(3,3)$ & $1(3,3)$ & $30(100)$ \\
\hline & $12 a 7 m-13 a$ & $1(3,3)$ & $3(10,0)$ & $13(43,3)$ & $7(23,4)$ & $6(20,0)$ & - & $30(100)$ \\
\hline & 13a1m-13a6m & - & - & $7(23,4)$ & $12(40,0)$ & $10(33,3)$ & $1(3,3)$ & $30(100)$ \\
\hline & $13 a 7 m-14 a$ & - & - & $5(16,7)$ & $13(43,3)$ & $11(36,7)$ & $1(3,3)$ & $30(100)$ \\
\hline TOTAL & & & & & & & & 464 \\
\hline
\end{tabular}

TABELA 3 - Distribuição da amostra de acordo com o estágio de calcificação, a idade e o gênero.

\begin{tabular}{|c|c|c|c|c|c|c|}
\hline gênero & idade & $\begin{array}{c}\text { estágio } 7 \\
n(\%)\end{array}$ & $\begin{array}{c}\text { estágio } 8 \\
n(\%)\end{array}$ & $\begin{array}{c}\text { estágio } 9 \\
n(\%)\end{array}$ & $\begin{array}{c}\text { estágio } 10 \\
\mathrm{n}(\%)\end{array}$ & $\begin{array}{l}\text { total } \\
\mathrm{n}(\%)\end{array}$ \\
\hline \multirow{8}{*}{ masculino } & $10 a-10 a 6 m$ & $13(52,0)$ & $9(36,0)$ & $3(12,0)$ & - & $25(100)$ \\
\hline & $10 a 7 m-11 a$ & $9(34,6)$ & $7(26,9)$ & $10(38,5)$ & - & $26(100)$ \\
\hline & $11 \mathrm{a} 1 \mathrm{~m}-11 \mathrm{a} 6 \mathrm{~m}$ & $3(10,0)$ & $9(30,0)$ & $18(60,0)$ & - & $30(100)$ \\
\hline & $11 a 7 m-12 a$ & - & $4(13,3)$ & $25(83,4)$ & $1(3,3)$ & $30(100)$ \\
\hline & $12 a 1 m-12 a 6 m$ & $1(3,3)$ & $5(16,6)$ & $22(73,4)$ & $2(6,7)$ & $30(100)$ \\
\hline & $12 a 7 m-13 a$ & $1(3,3)$ & $1(3,3)$ & $24(80,0)$ & $4(13,4)$ & $30(100)$ \\
\hline & 13a1m-13a6m & - & - & $24(80,0)$ & $6(20,0)$ & $30(100)$ \\
\hline & $13 a 7 m-14 a$ & - & - & $17(63,0)$ & $10(7,0)$ & $27(100)$ \\
\hline \multirow{8}{*}{ feminino } & $10 a-10 a 6 m$ & $4(15,4)$ & $11(42,3)$ & $11(42,3)$ & - & $26(100)$ \\
\hline & $10 a 7 m-11 a$ & $2(6,7)$ & $9(30,0)$ & $19(63,3)$ & - & $30(100)$ \\
\hline & $11 \mathrm{a} 1 \mathrm{~m}-11 \mathrm{a} 6 \mathrm{~m}$ & $2(6,7)$ & $7(23,3)$ & $21(70,0)$ & - & $30(100)$ \\
\hline & $11 a 7 m-12 a$ & $1(3,3)$ & $4(13,4)$ & $24(80,0)$ & $1(3,3)$ & $30(100)$ \\
\hline & 12a1m-12a6m & - & - & $27(90,0)$ & $3(10,0)$ & $30(100)$ \\
\hline & $12 a 7 m-13 a$ & - & - & $23(76,7)$ & $7(23,3)$ & $30(100)$ \\
\hline & 13a1m-13a6m & - & $1(3,3)$ & $18(60,0)$ & $11(36,7)$ & $30(100)$ \\
\hline & $13 a 7 m-14 a$ & - & - & $17(56,7)$ & $13(43,3)$ & $30(100)$ \\
\hline TOTAL & & & & & & 464 \\
\hline
\end{tabular}




\section{Relação entre os índices de maturação das vértebras cervicais e os estágios de calcificação dentária}

De acordo com Franchi, Baccetti e McNamara $\mathrm{Jr}^{10}{ }^{10}$ os índices de maturação das vértebras cervicais incluem características antes do pico, ou seja, durante a fase acelerativa de crescimento (IMVC 1 a 3), e características após o pico, ou seja, durante a fase desacelerativa de crescimento (IMVC 4 a 6). Portanto, o pico de crescimento puberal, ocorria, em média, entre os índices vertebrais 3 e $4^{10}$.

Analisando-se a frequência de distribuição da amostra de acordo com as variáveis estudadas, é possível verificar que, no gênero masculino, a fase acelerativa do SCP (IMVC 1 e 2) ocorreu com maior frequência até os 12 anos de idade. Nessa faixa etária, o segundo molar inferior já se encontrava no estágio 9 de Nolla na maioria da amostra, porém uma grande parcela ainda se encontrava nos estágios 7 e 8 . A partir dos 12 anos e 1 mês de idade, a maior parte da amostra já se mostrou em um nível mais avançado de maturação, alcançando o pico de crescimento (IMVC 3 e 4), embora uma pequena parte da amostra já tenha alcançado a fase desacelerativa de crescimento (IMVC 5 e 6). Em relação à calcificação do segundo molar inferior, este já se encontrava no estágio 9 de Nolla na maior parte da amostra, com uma pequena parte já se mostrando em estágio 10.

No gênero feminino, a maior parte da amostra até os 12 anos de idade se mostrou também na fase acelerativa do SCP, porém vários indivíduos já se encontravam no pico de crescimento. Nessa idade, o segundo molar inferior se encontrou no estágio 9 de Nolla na maioria da amostra. A partir dos 12 anos e 1 mês, observa-se que grande parte da amostra se encontrou no pico do SCP, já avançando para a fase desacelerativa do crescimento. Nesta fase, o segundo molar inferior se encontrou ainda no estágio 9 de Nolla, porém com uma grande parcela da amostra já em estágio 10.

Para verificar se, possivelmente, existiu associação entre os índices de maturação das vértebras cervicais e os estágios de calcificação dentária, optou-se por executar o coeficiente de contingência C. Para a realização desse teste, os índices de maturação das vértebras cervicais tiveram que ser agrupados em fases, uma vez que alguns índices não foram encontrados em quantidade suficiente para serem analisados estatisticamente. Para o agrupamento desses índices, obedeceu-se às observações de Franchi, Baccetti e McNamara Jr. ${ }^{10}$, dividindo-os em 3 fases, assim consideradas:

- $1^{\text {a }}$ fase: início do SCP - IMVC 1 e 2;

- $2^{a}$ fase: pico de crescimento puberal - IMVC 3 e 4 ;

- $3^{\text {a }}$ fase: final do SCP - IMVC 5 e 6.

Com a aplicação desse teste estatístico, obtevese como coeficientes de contingência $C$ os valores 0,37 e 0,32 para os gêneros masculino e feminino, respectivamente $(\mathrm{p}<0,05)$, mostrando que os estágios de calcificação do segundo molar inferior se correlacionaram estatisticamente com os índices de maturação das vértebras cervicais, em ambos os gêneros.

Um dado importante a ser considerado é que, em ambos os gêneros, a maturação das vértebras cervicais ocorreu em um período de tempo relativamente uniforme entre os grupos etários. Por outro lado, viu-se que a amostra estudada alcançou o estágio 9 de Nolla em um período de tempo menor que o período envolvido para mudar do estágio 9 para o estágio 10. Isso pode mostrar que, possivelmente, a finalização da formação dentária necessite de um período de tempo maior que o alcance da fase de pico de crescimento, o que pode sugerir que, mesmo encontrando associações entre essas duas variáveis, tal achado, quando considerado, pode ser um fator influenciador em alguns estudos que não encontrarem associações entre elas. Essa correlação também foi encontrada na literatura ${ }^{9,15,28}$. Peter ${ }^{26}$ propôs estudar a relação entre o SCP, os estágios de calcificação dentária e a idade cronológica em crianças de 7 a 15 anos de idade. A autora analisou também o segundo molar inferior, porém determinou as fases do SCP baseando-se na análise da 
curva de crescimento, obtida através da radiografia de mão e punho. Seus resultados demonstraram uma alta correlação entre as variáveis estudadas em ambos os gêneros, além de ter observado que o desenvolvimento dentário acompanhou as fases do SCP. Nota-se que seus resultados estão de acordo com os encontrados nesse estudo. Além disso, as cidades envolvidas nestes estudos (Fortaleza e São Luís, respectivamente) pertencem à mesma região do país, onde ambas as populações se comportaram de maneira semelhante.

A literatura demonstra essa correlação considerando também outros elementos dentários como referência. $\mathrm{O}$ desenvolvimento do canino inferior, por exemplo, tem demonstrado fortes associações com a maturidade esquelética ${ }^{7,16,23,24}$, sugerindo-se a possibilidade do uso dos estágios de calcificação dentária como indicadores do crescimento, sendo um método fácil, prático e econômico para o paciente $23,24,26$.

Entretanto, outros estudos não encontraram associações entre essas duas variáveis ${ }^{15,17,22,30}$. Segundo Dermijian et al. ${ }^{8}$, a baixa associação do desenvolvimento dentário com as maturidades esquelética, somática e sexual seria atribuída à diferença na escala ou à independência dos mecanismos que controlam esses eventos.

Assim, mesmo com inúmeros estudos abordando esse assunto, percebe-se que ainda permanecem divergências entre seus resultados, possivelmente pelas diferenças metodológicas existentes.
Outros fatores importantes que poderiam originar resultados divergentes seriam as variações interregionais (diferenças raciais, clima, nutrição, níveis socioeconômicos). Comparações do estado de uma amostra populacional contra padrões específicos de uma população de outra região poderiam superestimar o grau de retardo ou precocidade de desenvolvimento da amostra em estudo ${ }^{14,27,30}$.

É importante ressaltar a dificuldade encontrada em confrontar os resultados desse trabalho com a literatura, visto que ainda não foram encontrados estudos sobre este tipo de relação utilizando os índices de maturação das vértebras cervicais como método indicador das fases do SCP. Por isso, mais estudos utilizando esse método de análise da maturação óssea são recomendados, usando uma amostra mais ampla, em diferentes faixas etárias e analisando outros elementos dentários, para que resultados mais conclusivos possam ser obtidos.

\section{CONCLUSÕES}

Com base na literatura consultada e nos resultados obtidos neste estudo, foi observada uma correlação estatisticamente significativa entre os índices de maturação das vértebras cervicais e os estágios de calcificação do segundo molar inferior, em ambos os gêneros. Além disso, houve uma precocidade, tanto na maturação das vértebras cervicais quanto na calcificação do segundo molar inferior, no gênero feminino em relação ao gênero masculino.

\title{
Relationship between the cervical vertebrae maturation indicators and the dental calcification stages
}

\begin{abstract}
Aim: To evaluate the relationship between the different cervical vertebrae maturation indicators and the dental calcification stages, as well as to verify the existent differences among gender, in a sample of subjects with 10 to 14 years-old. Methods: The sample consisted of lateral cephalometric and panoramic radiographs of 464 subjects of both gender, classified in agreement with the cervical vertebrae maturation indicators described by Hassel and Farman (1995) and the calcification stages described by Nolla (1960). Results: The obtained results showed correlation among the studied variables $(p<0,05)$. Conclusions: Confirming data from the literature, a precocity was observed as much in the cervical vertebrae maturation indicators, as in the dental calcification stages for the females in relation to the males.
\end{abstract}

Keywords: Maturity. Cervical vertebrae. Teeth calcification. 


\section{REFERÊNCIAS}

1. BACCETTI, T.; FRANCHI, L.; McNAMARA JR., J. A. An improved version of the cervical vertebral maturation (CVM) method for the assessment of mandibular growth. Angle Orthod., Appleton, v. 72, no. 4, p. 316-323, Aug. 2002.

2. BACCETTI, T.; FRANCHI, L.; MCNAMARA JR., J. A. The cervical vertebral maturation method: some need for clarification. Am. J. Orthod. Dentofacial Orthop., St. Louis, v. 123, no. 1, p. 19A-20A, Jan. 2003. Disponível em: <http://www2. us.elsevierhealth.com/scripts/om.dll/serve?action=searchDB\&s earchDBfo>. Acesso em: 12 jan. 2004.

3. BACCETTI, T.; FRANCHI, L.; McNAMARA JR., J. A. The cervical vertebral maturation (CVM) method for the assessment of optimal treatment timing in dentofacial orthopedics. Semin. Orthod., Philadelphia, v. 11, p. 119-129, 2005.

4. BIOESTAT 3.0 [programa de computador]. Versão 3.0. Belém: Sociedade Civil: MCT - CNPQ, 2003.

5. CARVALHO, A. A. F. Estimativa de valores médios de crescimento em crianças com idade cronológica variando de 84 a 131 meses. Rev. Odontol. UNESP, Marília, v. 22, n. 2, p. 293-301, jul./dez. 1993.

6. CARVAlho, A. A. F.; CARVAlHO, A.; SANTOS-PINTO, M. C. Estudo radiográfico do desenvolvimento da dentição permanente de crianças brasileiras com idade cronológica variando entre 84 e 131 meses. Rev. Odontol. UNESP, Marília, v. 19, n. 1, p. 31-39, 1990.

7. COUTINHO, S.; BUSCHANG, P. H.; MIRANDA, F. Relationships between mandibular canine calcification stages and skeletal maturity. Am. J. Orthod. Dentofacial Orthop., St. Louis, v. 104, no. 3, p. 262-268, Sept. 1993.

8. DERMIJIAN, A. et al. Interrelationships among measures of somatic, skeletal, dental and sexual maturity. Am. J. Orthod., St. Louis, v. 88, no. 5, p. 433-438, Nov. 1985.

9. ETO, L. F. Correlação entre os estágios de mineralização dentária na arcada inferior e a idade esquelética. 2001. Dissertação (Mestrado)-Faculdade de Odontologia, Pontifícia Universidade Católica de Minas Gerais, Belo Horizonte, 2001.

10. FRANCHI, L.; BACCETTI, T.; McNAMARA JR., J. A. Mandibular growth as related to cervical vertebrae maturation and body height. Am. J. Orthod. Dentofacial Orthop., St. Louis, v. 18, no. 3, p. 335-340, Sept. 2000.

11. GARCIA-FERNANDEZ, P. et al. The cervical vertebrae as maturational indicators. J. Clin. Orthod., Boulder, v. 31, no. 4, p. 221-225, Apr. 1998.

12. GUZZI, B. S. S.; CARVALHO, L. S. Estudo da maturação óssea em pacientes jovens de ambos os sexos através de radiografias de mão e punho. Ortodontia, São Paulo, v. 33, n. 3, p. 49-58, set./dez. 2000

13. HAITER NETO, F. Análise comparativa manual e computadorizada da estimativa da idade óssea pelo índice de Eklöf \& Ringertz. 1995. Tese (Doutorado)-Faculdade de Odontologia de Bauru, Universidade de São Paulo, Bauru, 1995.

14. HASSEL, B.; FARMAN, A. G. Skeletal maturation evaluation using cervical vertebrae. Am. J. Orthod. Dentofacial Orthop., St. Louis, v. 107, no. 1, p. 58-66, Jan. 1995.

15. HELM, S. Relationship between dental and skeletal maturation in Danish schoolchildren. Scand. J. Dent. Res., Copenhagen, v. 98, no. 4, p. 313-317, Aug. 1990.

16. KRAILASSIRI, S. et al. Relationships between dental calcification stages and skeletal maturity indicators in Thai individuals. Angle Orthod., Appleton, v. 72, no. 2, p. 155-166, Apr. 2002.

17. KULLMAN, L.; JOHANSON, G.; AKESSON, L. Root development of the lower third molar and its relation to chronological age. Swed. Dent. J., Jonkoping, v. 16, no. 4, p. $161-167,1992$.

18. LAMPARSKI, D. G. Skeletal age assessment utilizing cervical vertebrae. 1972. Dissertation (Master of Dental Science)-Faculty of the School of Dental Medicine, University of Pittsburgh, Pittsburgh, 1972.
19. LELES, C. R. Princípios de bioestatística. In: ESTRELA, C. Metodologia científica: ensino e pesquisa em Odontologia. São Paulo: Artes Médicas, 2001. cap. 14, p. 275-306.

20. LIMA, A. A. S. J. Avaliação do surto de crescimento puberal através da análise radiográfica das vértebras cervicais. 2004. Dissertação (Mestrado)-Universidade Federal do Maranhão, São Luís, 2004.

21. MITO, T.; SATO, K.; MITANI, H. Predicting mandibular growth potential with cervical vertebral bone age. Am. J. Orthod. Dentofacial Orthop., St. Louis, v. 124, no. 2, p. 173-177, Aug. 2003.

22. MORAES, M. E. L. Surto de crescimento puberal: relação entre mineralização dentária, idade cronológica, idade dentária e idade óssea: método radiográfico. 1997. Tese (Doutorado)Faculdade de Odontologia de São José dos Campos, Universidade Estadual Paulista "Júlio de Mesquita Filho", São José dos Campos, 1997

23. NADLER, G. L. Earlier dental maturation: fact or fiction? Angle Orthod., Appleton, v. 68, no. 6, p. 535-538, Apr. 1998.

24. NAVARRO, M. T. A. Relación entre el desarrollo esquelético y los estados de calcificación del canino inferior. 1996. Trabalho de Conclusão de Curso (Especialização)-Faculdade de Odontologia, Escola Colombiana de Medicina, Santa Fé de Bogotá, 1996.

25. NOLLA, C. M. The development of the permanent teeth. J. Dent. Child., Fulton, v. 27, p. 254-266, 1960.

26. PETER, E. A. Relação entre surto de crescimento puberal, desenvolvimento dentário e idade cronológica em crianças e adolescentes na faixa etária de 7 a 15 anos. 2003. Dissertação (Mestrado)-Faculdade de Odontologia de Bauru, Universidade de São Paulo, Bauru, 2003.

27. SANTOS, S. C. B. N.; ALMEIDA, R. P. Estudo comparativo de dois métodos de avaliação da idade esquelética utilizando telerradiografias em norma lateral e radiografias carpais. Ortodontia, São Paulo, v. 32, n. 2, p. 33-45, maio/ago. 1999.

28. SIERRA, A. M. Assessment of dental and skeletal maturity. Angle Orthod., Appleton, v. 57, no. 3, p. 194-208, July 1987.

29. SILVA, G. C. H. Avaliação do surto de crescimento puberal através do exame clínico e radiográfico dos estágios de calcificação do segundo pré-molar inferior. 1992. Dissertação (Mestrado)-Faculdade de Odontologia, Universidade Federal do Rio de Janeiro, Rio de Janeiro, 1992.

30. SILVEIRA, A. M. et al. Facial growth during adolescence in early, average and late maturers. Angle Orthod., Appleton, v. 62 , no. 3 , p. $185-190$, Mar. 1992.

Enviado em: julho de 2006 Revisado e aceito: março de 2008

Endereço para correspondência

Clarissa Lopes Vieira

Rua Rio Anil, Quadra 7, Condomínio Mar del Plata,

Casa 25, Recanto dos Vinhais

CEP: 65.070-018 - São Luís/MA

E-mail: cleovi@gmail.com 